\title{
Optimization of SIMD Programs with Redundant Computations
}

\author{
Jörn Eisenbiegler \\ Institut für Programmstrukturen und Datenorganisation, \\ Universität Karlsruhe, 76128 Karlsruhe, Germany \\ eiseneipd.info.uni-karlsruhe.de
}

\begin{abstract}
This article introduces a method for using redundant computations in automatic compilation and optimization of SIMD programs for distributed memory machines. This method is based on a generalized definition of parameterized data distributions, which allows an efficient and flexible use of redundancies. An example demonstrates the benefits of this optimization method in practice.
\end{abstract}

\section{Introduction}

edundant computations, i.e. computations that are done on more than one prossor, can improve the performance of parallel programs on distributed memory machines [2]. In the examples given in section 5 , the execution time is improved by nearly one magnitude. Optimization methods (e.g. $[5,7]$ ) which use redundant computations have two major disadvantages: First the task graph of the program has to be investigated node per node. Second the communication costs have to be estimated without knowledge about the final data distribution.

To avoid these disadvantages, other methods as for example proposed in $[3,4$, 8] use parameterized data distributions. But, the data distributions given there do not allow redundant computations in a more flexible way than "all or nothing". Therefore, this article introduces a scheme for defining parameterized data distributions, which allow the efficient and flexible use of redundant computations.

Based on these definitions, this article describes a method for optimizing SIMD programs for distributed memory systems. This method is divided into three steps. As in [1], the first step uses heuristics (as described in $[3,4,8]$ ) to propose locally good data distributions. However, these distributions are not used on the level of loops but on the level of parallel assignments. In the second step, additional data distributions (which lead to redundant computations) are calculated which connect subsequent parallel assignments in a way such that no communication is needed between them. The last step selects a globally optimal proposition for each parallel assignment. In contrast to [1], an efficient algorithm is described instead of 0-1 integer programming. The benefits of this optimization method are shown by the example of numerically solving of a differential equation. 


\section{Parameterized Data Distributions}

In order to define parameterized data distributions, a definition for data distributions is required. Generally, a data element can exist on any number of processors and a processor can hold any number of data elements. Therefore, distributions have to be defined as relations between elements and processors:

Definition 1 (data distribution). A data distribution is a set $\mathrm{D} \subseteq \mathrm{N}_{0} \times \mathrm{N}_{0}$ of tuples. $(p, i) \in \mathrm{D}$ iff element $i$ is valid on processor $p$, $i$. e. element $i$ has the correct value on processor $p$.

As an example, assume a machine with $P=4$ processors and an array $a$ with 15 elements. The set $D=\{(0,0),(0,1),(0,2),(0,10),(0,11),(0,12),(0,13)$, $(0,14),(1,1),(1,2),(1,3),(1,4),(1,5),(1,13),(1,14),(2,4),(2,5),(2,6),(2,7)$, $(2,8),(3,7),(3,8),(3,9),(3,10),(3,11)\}$ is a data distribution where the elements $1,2,4,5,7,8,10,11,13,14$ are valid on exactly two out of four processors.

For practical purposes in a compiler or for hand optimization of non-trivial programs, such data structures are too large. Therefore, parameterized data distributions are used. Each combination of parameters represents a certain data distribution. The correlation between the parameters and the data distributions they represent is the interpretation, i.e. an interpretation of a parameterized data distribution maps parameters to a data distribution as defined in Definition 1:

Definition 2 (parameterized data distribution).

A parameterized data distribution space is a pair $(\mathrm{S}, I)$, where $\mathrm{S}$ is an arbitrary set and $I: \mathrm{S} \rightarrow 2^{\mathrm{N}_{0} \times \mathrm{N}_{0}}$ is interpretation function. Every $\mathrm{P} \in \mathrm{S}$ is a parameterized data distribution, i.e. $(p, i) \in I(\mathrm{P})$ iff element $i$ is valid on processor $p$.

Usually, the set $S$ (the set of parameters) is a set of tuples whose elements describe the number of processors, block length etc. for each array dimension. In order to carry on with the example, regard the data distribution space $\left(\mathrm{N}_{0}^{3}, I\right)$ with interpretation $I$ :

$$
I(b, a, r):=\{(p, i) \mid a+k b P+p b-r \leq i<a+k b P+(p+1) b+r, k \in Z\}
$$

The parameter $b$ is the block size, the parameter $a$ the alignment, and the parameter $r$ describes redundancy. In this data distribution space, the data distribution $\mathrm{D}$ described above is parameterized with $(3,-1,1)$, because $I(3,-1,1)=\mathrm{D}$. Note, that the correlation between processors and data elements is still relational, even if the interpretation $I$ of the parameters is a function. Therefore definition 2 is a generalization of former definitions (e.g. [3, 8]).

The subset relation defines a semi-ordering for data distributions. This semiordering can be transferred to parameterized data distributions:

Definition 3 (semi-ordering). Let (S, I) be a parameterized data distribution space, $\mathrm{P}_{1}, \mathrm{P}_{2} \in \mathrm{S}: \mathrm{P}_{1} \leq \mathrm{P}_{2}$, iff $I\left(\mathrm{P}_{1}\right) \subseteq I\left(\mathrm{P}_{2}\right)$.

Theorem 1. Let $(\mathrm{S}, I)$ be a parameterized data distribution space, $\mathrm{P}_{1}, \mathrm{P}_{2} \in \mathrm{S}$. There are no communication costs for changing the distribution of an array from $\mathrm{P}_{1}$ to $\mathrm{P}_{2}$, iff $\mathrm{P}_{1} \geq \mathrm{P}_{2}$. 
Proof. Let $\mathrm{P}_{1} \geq \mathrm{P}_{2}$. Then, every data element which is valid on a processor in $\mathrm{P}_{1}$ is also valid their in $\mathrm{P}_{2}$, since $I\left(\mathrm{P}_{1}\right) \supseteq I\left(\mathrm{P}_{2}\right)$. Therefore, no data elements have to be transferred and no communication costs occur. If $P_{1} \geq P_{2}$, there is at least one data element valid on a processor in $I\left(\mathrm{P}_{2}\right)$ which is not valid their in $I\left(\mathrm{P}_{1}\right)$. This data element has to be transferred generating communication costs.

The data distribution state of a program is described by the data distribution of each array in the program. This state is defined by a data distribution function, which maps arrays to parameterized data distributions.

Definition 4 (data distribution functions). Let $I D$ be the name space of a program and $(\mathrm{S}, I)$ be a parameterized data distribution space. Then, a function $f: I D \rightarrow \mathrm{S}$ is called a data distribution function. Let $f_{1}$ and $f_{2}$ be two data distribution functions. $f_{1} \leq f_{2}$ iff $\forall x \in I D: f_{1}(x) \leq f_{2}(x)$.

\section{Parallel Array Assignments}

With the definitions given in section 2 , it is possible to distinguish between the input and output data distribution of a parallel assignment. As an example, regard the data distribution space described above and the parallel assignment

par $\mathrm{i}$ in $[1, n-1] x[i]:=x[i-1]+x[i]+x[i+1]$;

If the output data distribution of $x$ is to be $(3,-1,0)$, the input data distribution for $x$ must be at least $(3,-1,1)$. This distinction is not possible with former definitions of parameterized data distributions, since it is not possible to describe the input data distribution there.

In order to optimize data distributions for arrays, the execution of an SIMDprogram can now be seen as a sequence of array assignments. Every assignment has an input and an output data distribution function. Between two assignments the data distribution state has to be changed from the output data distribution of the first to a data distribution which is larger or equal to the input data distribution of the second assignment. Note that there is no difference between the remapping of data and getting the necessary input for an assignment. In this article, the overlapping of communication and computation is not examined. However, this might be used for further optimization.

\section{Optimization}

\subsection{Proposition}

In the first step of the optimization, heuristics propose locally good data distributions for each parallel assignment. This paper does not go into detail about searching locally good data distributions, since this problem is well-investigated (e.g. $[3,8])$. It is allowed to propose more than one data distribution for one parallel assignment using more than one proposition method to exploit the benefits of different heuristics. 
The methods mentioned above only compute the output data distributions for the array written in the assignment. Therefore the corresponding input and output data distribution functions have to be calculated. If there are no data elements of an array needed in a data distribution function, this array is declared as undefined. As the result of this step, each parallel assignment is applied with several proposals of pairs of input and output data distribution functions.

\subsection{Redundancy}

In the second step of optimization, additional propositions are introduced which connect subsequent parallel assignments in a way such that no communication is necessary. Let $a_{1}$ and $a_{2}$ be two subsequent parallel assignments and $\left(i_{1}, o_{1}\right)$ and $\left(i_{2}, o_{2}\right)$ pairs of input and output distribution functions proposed for $a_{1}$ and $a_{2}$, respectively. Let $n$ be the array written in $a_{1}$ and $d_{1} \cup d_{2}$ be a parameterized data distribution which is larger than $d_{1}$ and $d_{2}$. First, $\left(i_{1}^{\prime}, o_{1}^{\prime}\right)$ is set to the pair of distribution functions corresponding to the proposal $i_{2}(n) \cup o_{2}(n)$ for $n$ in $a_{1}$ (as above). Hence, no communication is needed to redistribute $n$ from $o_{1}^{\prime}(n)$ to $i_{2}(n)$. Second, to avoid communication for other arrays, $\left(i_{1}^{*}, o_{1}^{*}\right)$ is set to

$$
o_{1}^{*}(x)=\left\{\begin{array}{ll}
o_{1}^{\prime}(x) \cup i_{2}(x) & x \neq n \\
o_{1}^{\prime}(x) & x=n
\end{array} \quad i_{1}^{*}(x)= \begin{cases}i_{1}^{\prime}(x) \cup i_{2}(x) & x \neq n \\
i_{1}^{\prime}(x) & x=n\end{cases}\right.
$$

As a result of this calculation, $o_{1}^{*}$ is larger than $i_{2}$, and therefore no communication is needed to redistribute from $o_{1}^{*}$ to $i_{2}$. However, in most cases some data elements have to be calculated redundantly on more than one processor for $a_{1}$.

The method can be applied to all combinations of proposals for $a_{1}$ and $a_{2}$. Especially, it can be applied to cost tuples for $a_{2}$ which were themselves proposed this way. This leads to (potential) sequences in the program where no communication is needed. At the end of this step, again all parallel assignments are applied with several pairs of input and output data distribution functions.

\subsection{Configuration}

The configuration step tries to select the globally optimal data distribution for each parallel assignment from the local proposals. If the program is not oblivious (see [5]), it can not be guaranteed that the optimal selection is found due to the lack of compile time knowledge.

In order to find an optimal solution for arbitrary programs, the configuration method by Moldenhauer [6] is adopted. The proposals for the parallel assignments are converted to cost tuples of the form $(i, c, o)$ where $i$ and $o$ are the proposed input and output distribution functions and $c$ are the costs for computing this parallel assignment with the proposed distributions. To find an optimal solution for the whole program, these sets of cost tuples are combined to sets of cost tuples for larger program blocks.

Let $a_{1}$ and $a_{2}$ be two subsequent program blocks and $M_{1}$ and $M_{2}$ be the sets of cost tuples proposed or calculated for these blocks. If $a_{1}$ is executed 
with $\left(i_{1}, c_{1}, o_{1}\right)$ and $a_{2}$ is exccuted with $\left(i_{2}, c_{2}, o_{2}\right)$ then $a_{1} ; a_{2}$ is executed with $(i, c, o)=\left(i_{1}, c_{1}, o_{1}\right) o_{t}\left(i_{2}, c_{2}, o_{2}\right)$ where

$$
\begin{aligned}
& i(x)=\left\{\begin{array}{l}
i_{1}(x) \text { if } i_{1}(x) \text { or } o_{1}(x) \text { is defined } \\
i_{2}(x) \text { else if } i_{2}(x) \text { is defined }
\end{array}\right. \\
& c=c_{1}+c_{2}+\text { redistribution_costs }\left(o_{1}, i_{2}\right) \\
& o(x)=\left\{\begin{array}{l}
o_{2}(x) \text { if } o_{2}(x) \text { is defined } \\
o_{1}(x) \text { else }
\end{array}\right.
\end{aligned}
$$

For further combination with other program blocks, the cost tuples with minimal costs for all combinations of input and output data distribution functions which can occur using the proposals are calculated, i.e. the set of cost tuples $M:=$ $M_{1} \circ M_{2}$ for the sequence $a_{1} ; a_{2}$ is

$$
\begin{aligned}
M:=M_{1} \circ M_{2}:= & \{(i, c, o) \mid(i, c, o) \text { is cost-minimal in } \\
& \left.\left\{\left(i, c^{*}, o\right)=\left(i_{1}, c_{1}, o_{1}\right) o_{t}\left(i_{2}, c_{2}, o_{2}\right) \mid\left(i_{k}, c_{k}, o_{k}\right) \in M_{k}\right\}\right\}
\end{aligned}
$$

Rules for other control constructs can be given analogously and are omitted in this article. Using these rules, the set of cost tuples for the whole program can be calculated. In the set for the whole program the element with minimal costs is selected. For oblivious programs it represents the optimal selection from the proposals. For non-oblivious programs heuristics have to be used for the combination of the sets. To obtain the optimal proposals for each parallel assignment, the origin of the tuples has to be remembered during the combination step.

This configuration problem is not in APX, i.e. no constant approximation can be found. Accordingly, the algorithm described above is exponential. But in contrast to the solution proposed in [1], it is only exponential in the number of array variables simultaneously alive. Since this number is small in real applications the method is feasible.

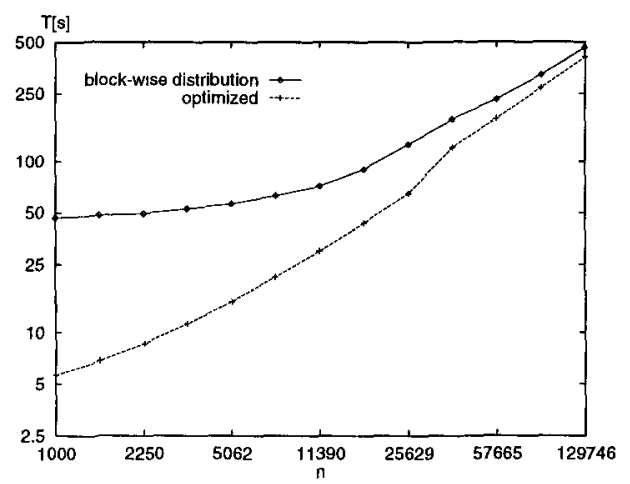

Fig. 1. Solving a differential equation 


\section{Practical Results}

The optimization method described above is implemented in a prototype compiler. This section shows the optimization results compared to results that can be achieved if no redundancy is used and data distribution is not done for parallel assignments but for loops only. All measurements were done on an 8 processor Parsytec PowerXplorer.

Figure 1 shows the runtimes for solving a differential equation in $n$ points. If no redundant computations are allowed, a blockwise distribution is optimal. Therefore the optimized program is compared to a version using blockwise distribution. Especially for small input sizes, the optimized program is nearly one magnitude faster than the blockwise distributed version.

\section{Conclusion and Further Work}

This article introduces a formal definition for parameterized data distributions that allow redundancies. This makes it possible to transfer the concept of redundant computations to the world of parameterized data distributions such that compilers can use redundant computations efficiently.

Based on these definitions, a method for optimizing SIMD programs on distributed systems is given. This method allows combining several local optimization methods for computing local proposals. Furthermore, redundant computations are generated to save communication. For oblivious programs the method selects the optimal proposals for each array assignment. The practical results show a large gain in performance.

In the future different data distribution spaces, i.e. parameters and their interpretations, have to be examined to select suitable ones. Additionally, optimal redistribution schemes for these data distribution spaces should be selected.

\section{References}

1. R. Bixby, K. Kennedy, and U. Kramer. Automatic data layout using 0-1 integer programming. In M. Cosnard, G. R. Gao, and G. M. Silberman, editors, Parallel Architecture and Compilation Techniques (A-50). IFIP, Elsevier Science B.V. (North-Holland), 1994.

2. J. Eisenbiegler, W. Löwe, and A. Wehrenpfennig. On the optimization by redundancy using an extended LogP model. In International Conference on Advances in Parallel and Distributed Computing (APDC'97), pages 149-155. IEEE Computer Society Press, March 1997.

3. M. Gupta. Automatic Data Partitioning on Distributed Memory Multicomputers. $\mathrm{PhD}$ thesis, Department of Computer Science, University of Illinois at UrbanaChampaign, 1992.

4. P. Z. Lee. Efficient algorithms for data distribution on distributed memory parallel computers. IEEE Transactions on Parallel and Distributed Systems, 8(8):825 - 839, August 1997. 
5. W. Löwe, W. Zimmermann, and J. Eisenbiegler. Optimization of parallel programs with expensive communication. In EUROPAR '96, Parallel Processing, volume 1124 of Lecture Notes in Computer Science, pages 602-610, 1996.

6. H. Moldenhauer. Kostenbasierte Konfigurierung für Programme und SW-Architekturen. Logos Verlag, Berlin, 1998. Dissertation, Universität Karlsruhe.

7. C. Papadimitriou and $M$. Yannakakis. Towards an architecture-independent analysis of parallel algorithms. SIAM Journal on Computing, 19(2):322 - 328, 1990.

8. M. Philippsen. Optimierungstechniken zur Übersetzung paralleler Programmiersprachen. Number 292 in VDI Fortschritt-Berichte, Reihe 10: Informatik. VDIVerlag GmbH, Düsseldorf, 1994. Dissertation, Universiät Karlsruhe. 